\title{
Vehicle to Vehicle Communication using Light Fidelity
}

\author{
T. N. Prabhu \\ Assistant Professor \\ Sri Ramakrishna Engineering \\ College
}

\author{
Adharsh M. \\ UG Student \\ Sri Ramakrishna Engineering \\ College
}

\author{
Ashok Kumar M. \\ UG Student \\ Sri Ramakrishna Engineering \\ College
}

\author{
Gokul Krishna M. \\ UG Student \\ Sri Ramakrishna Engineering College
}

\author{
Dhayanithi G. \\ UG Student \\ Sri Ramakrishna Engineering College
}

\begin{abstract}
Intelligent Transport System(ITS) are advanced applications that are used to provide various innovative services to facilitate road safety and traffic management, Vehicular communication is an advance technology that can be used in ITS. Vehicle-to-Vehicle (V2V) communication system using the emerging wireless system provides early warning signals to reduce road accidents and congestions. To improve the safety of the users a cooperative driving is proposed it also helps to improve the efficiency by enabling vehicles to communicate accident related messages with each other. Cooperative driving can also be advantageous in improving the safety of the neighborhood. It assists and help driver to take proper decision and avoid collision and congestion. In this paper design and result of vehicle to vehicle communication using Li-Fi(Light Fidelity), is presented .The proposed use of Li-Fi Technology in this paper comprises mainly of Light Emitting Diode(LED) bulbs as a means of connectivity by sending data through optical spectrum as an optical wireless medium for signal propagation. In fact, the usage of LED eliminates the need of complex wireless networks and protocols. A small scale prototype of vehicle to vehicle communication system using Light fidelity is presented.
\end{abstract}

\section{Keywords}

Intelligent Transport System, Light Emitting Diode, Visible light communication, Photodiode, Vehicle-to-Vehicle communication

\section{INTRODUCTION}

Unfortunately, most counties in the world has an alarming record in number of death/disability due to tremendous number of accident. Accidents are occurred because of unawareness of the people. Researchers [1] found that $57 \%$ of accidents where due to solely driver factors, which include his behavior, decision making ability, reaction speed and alertness.

The studies [1] show that the accidents can be avoided if driver was provided with warning message few seconds before so that, they can take some alternative route or be cautious to avoid traffic congestion or accidents[1]. The vehicular adhoc network was adopted to mimic the adhoc nature of highly dynamic network. In this network two vehicles can communicate with each other.

For Vehicle safety a new technique can be created. VANET Communication is classified into two different types Vehicle to Vehicle communication and Vehicle to Infrastructure Communication. The vehicle to vehicle communication is a communication between two vehicles (i.e.) one hop communication [4], such as car to car communication. The vehicle to Infrastructure communication is communication between vehicle and road side Infrastructure. It acts as a multi hop communication. The vehicle to vehicle communication is a system designed to transfer basic safety related with vehicles to provide warning to drivers concerning accidents. The main objective of this system is to alert drivers when he closes to front vehicle. The communication between the vehicles takes place by means of LI-FI.

The distance between two vehicles is measured using Ultrasonic sensor. The microcontroller controls the entire circuit and is programmed to notify the driver with a message when the vehicle comes within the Line of sight [3]. There are several obstacles that hinder the safety while driving. The vehicle such as car or buses may break down in middle of the road especially during the night time these becomes a serious obstacles mainly in highways were the roads are not lighted. The vehicle coming behind may not judge the stationary vehicle and may cause accident; the vehicle coming behind may hit hardly to the back of stationary vehicle and may lead to greater damage. Many scenarios were considered for the design of the system.

\subsection{First Scenario}

Vehicle1 slows down the speedometer senses the speed if lower than the previous speed an Ultrasonic Sensor attached in the bonnet using Doppler-effect is made to sense continuously. When the distance between the two vehicles decreases a warning message is transferred to back vehicle using the transmitter attached in the front vehicle it is received by the photo-detector attached to the back vehicle so he can take necessary steps to ensure that collision is avoided.

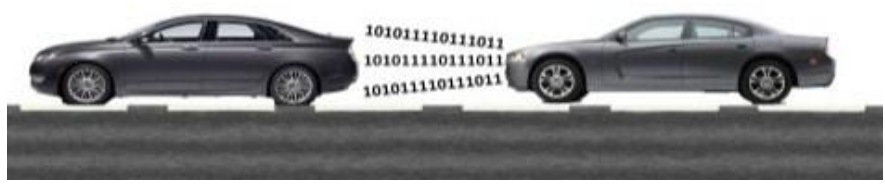

Fig1. First Scenario of Vehicle to Vehicle Communication Using VLC

\subsection{Second Scenario}

The major reasons for accidents are due to the negligence of the driver who might be under the influence of alcohol or might be sleeping while driver[6]. The vehicles are interfaced with an Alcohol as well as an Eye-blink sensor to monitor driver. If the driver is under the influence of alcohol or is sleeping this information is transferred to vehicle within the line of sight so vehicle can speed up or allow the vehicle to go ahead without causing any damage. 


\section{$\Rightarrow$ Vehicle to Vehicle}

\section{Fig2. Second Scenario of Vehicle to Vehicle Communication Using VLC}

The organization of paper is as follows section 2 provides information about the system design. The results and experimental setup are discussed in section 3 . The conclusion and future scope is discussed in section 4 .

\section{SYSTEM DESIGN}

The system is designed for two vehicles. The Vehicle Module (VM) is embedded with the vehicle so acts as moving nodes. It is responsible for communicating with vehicles and also the display the message received from vehicles. It consists of different sensors, microcontroller, Light emitting diode and photo diode to retrieve data. The vehicle module has many different features such as high performance, architecture simplicity, cost sensitivity and ultra-low power consumption. It consists of two major units Trans receiver Unit and a Control Unit. The Trans receiver unit is responsible for transferring data between two vehicles and the control unit is responsible for controlling the device.

\subsection{Visible Light Communication}

Visible light communication (VLC) is a data communications variant which uses visible light between 400 and $800 \mathrm{THz}$ (780-375 nm). VLC is a subset of optical wireless communications technologies. The technology uses fluorescent lamps (ordinary lamps, not special communications devices) to transmit signals at $10 \mathrm{Kbit} / \mathrm{s}$, or LEDs for up to $500 \mathrm{Mbit} / \mathrm{s}$. Low rate[vague] data transmissions at 1 and 2 kilometers $(0.6$ and $1.2 \mathrm{mi})$ were demonstrated. RONJA achieves full Ethernet speed (10 Mbit/s) over the same distance thanks to larger optics and more powerful LEDs. Specially designed electronic devices generally containing a photodiode receive signals from light sources, although in some cases a cell phone camera or a digital camera will be sufficient[6]. The image sensor used in these devices is in fact an array of photodiodes (pixels) and in some applications its use may be preferred over a single photodiode. Such a sensor may provide either multi-channel communication (down to 1 pixel $=1$ channel) or a spatial awareness of multiple light sources.

\subsection{Sensing Devices}

The Ultrasonic sensor consists of an ultrasonic transmitter and receiver. Ultrasonic sensor transmits and receives ultrasonic signal. It works on the Doppler Effect. The transmitter transmits the signal in one direction then signal is reflected back and received by the receiver. The distance between the object is measured by the total time taken by the signal to transmit and receive back.

The Alcohol Sensor consists of a tin oxide and a heating element inside a tubular casting. The Ethyl alcohol present in the breath is oxidized into acetic acid passing through heating element, which in turn reduces the resistance. Using external load resistance the resistance is converted into suitable voltage to signify alcohol content.

The Eye Blink Sensor uses IR sensor to check if person is sleeping or not. An IR transmitter sends IR signals to eyes and IR receiver is used to receive the signal reflected from eyes. The Logic high IR signal received signifies driver is sleeping and low IR signal signifies driver is brisk and active.

The proposed system is designed to work based on the following algorithm:

\section{Algorithm 1: Proposed System Algorithm}

Step 1: Initialize the LCD, UART 9600, and Li-Fi module.

Step 2: Activate the entire sensor and collect its data from the sensor and transfer it to the controller.

Step 3: Process all the data and LED will communicate.

Step 4: Read the Ultrasonic sensor data. Store and check the data.

Step 5: If the distance is less than the threshold value a warning message is transferred to the vehicle behind

Step 6: If the driver is intoxicated or is under influence of sleep a buzzer is activated to inform the drivers to take precautionary steps.

The block diagram for the proposed system is shown in the figure:

\subsection{Application Challenges}

The several challenges were considered in designing the proposed system i.e. processing delay, Line of sight Limitation. This Scenario can be better explained by presenting an analogy of three vehicles (namely A, B, C) travelling in a lane. When A suddenly applies break not only $\mathrm{A}$ and $\mathrm{B}$ are in danger but $\mathrm{C}$ also is in danger due to aforementioned reason.

\section{i. Large processing time}

The reaction time of the driver is more [i.e.] when driver A applies a brake after seeing the brake light the driver B applies brake; the range of delay is very high. This delay must be avoided in transmission of warning messages.

\section{ii. Line of sight Limitation}

The vehicle B might only see brake light of the front vehicle $\mathrm{A}$ while vehicle $\mathrm{C}$ may not be see the brake light of A due to line of sight limitation. 


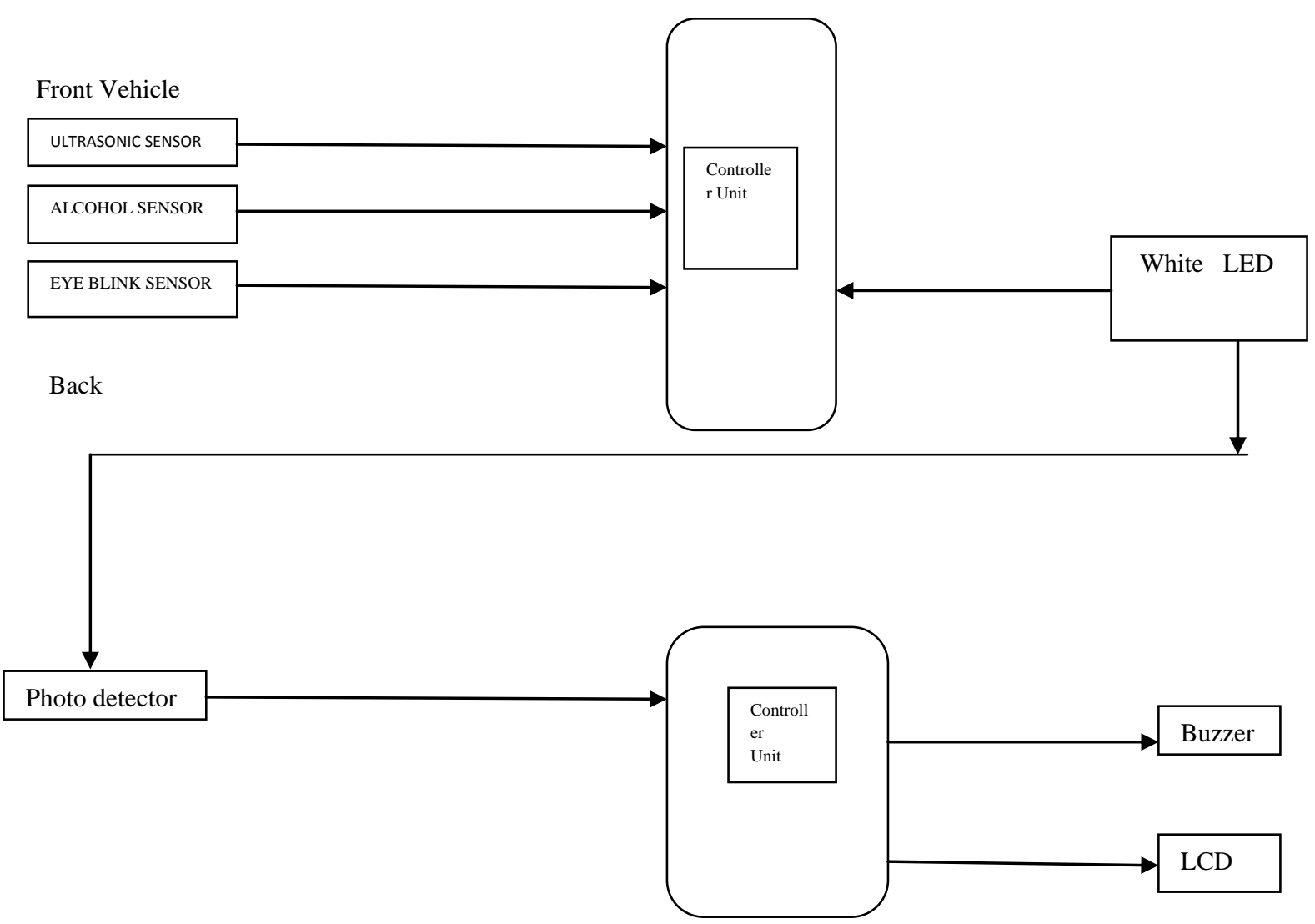

Fig3.Block diagram for Vehicle to Vehicle communication

\section{EXPERIMENTAL SETUP AND RESULTS}

The top view of the front vehicle and back vehicle is presented. The top view presents the various sensors interfaced with the control unit.

The Top view of the front vehicle consists of a buzzer and a LCD monitor to notify the driver with a warning message. The Photodetector is used in front vehicle to receive the data transmitted by the LED.

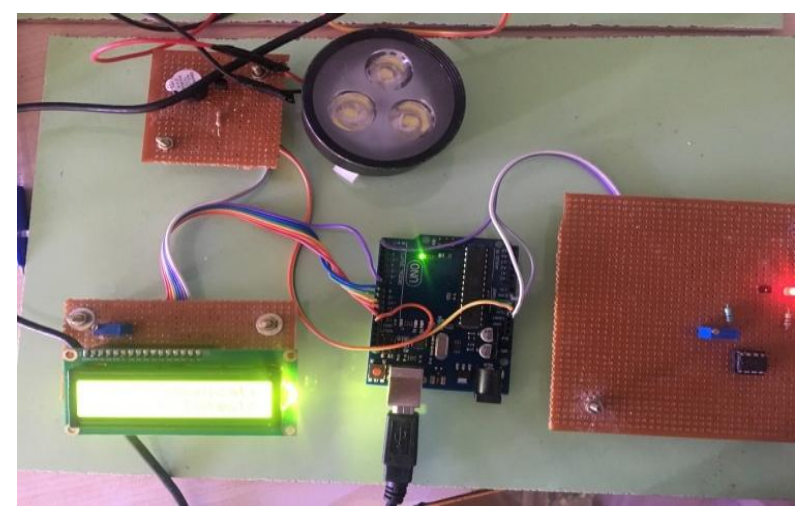

Fig4.Top View of Front Vehicle

The Back vehicle consists of Ultrasonic sensor to measure the distance between the two vehicles. An Eye-Blink sensor is used to check if the driver is sleeping while driving and alcohol sensor is interfaced with control unit to find if the driver is intoxicated by alcohol and use the LED to transmit the safety related message so that the front vehicle can be notified.

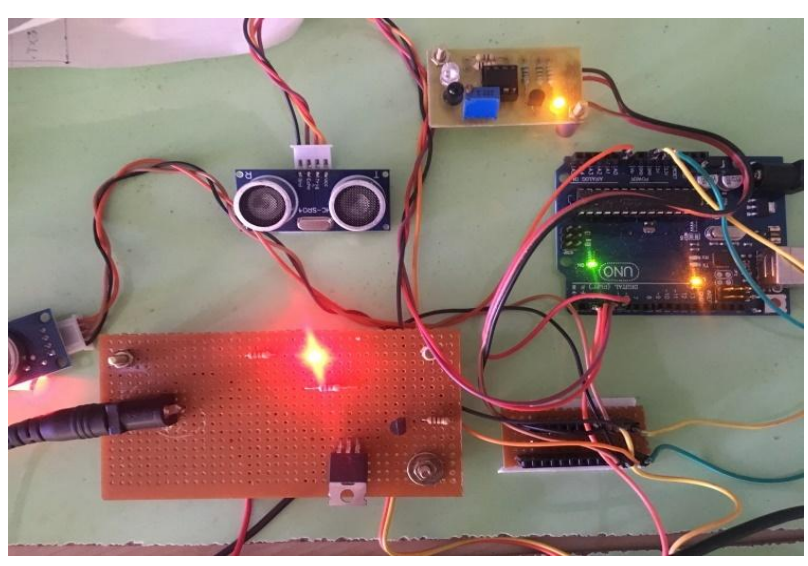

Fig5. Top View of Back Vehicle

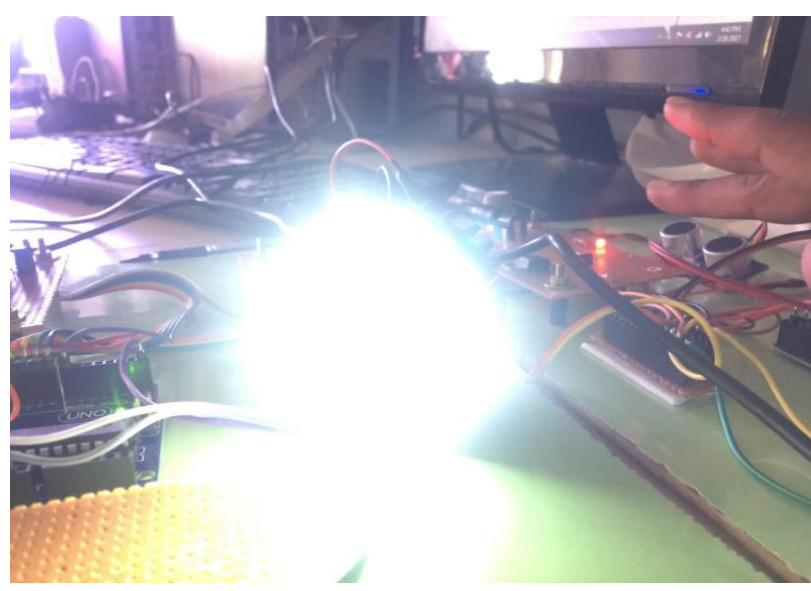

Fig6. LED Transmitting data 
The LED transmits the data when the ultrasonic sensor detects an obstruction, when the driver is under the influence of alcohol and is sleeping

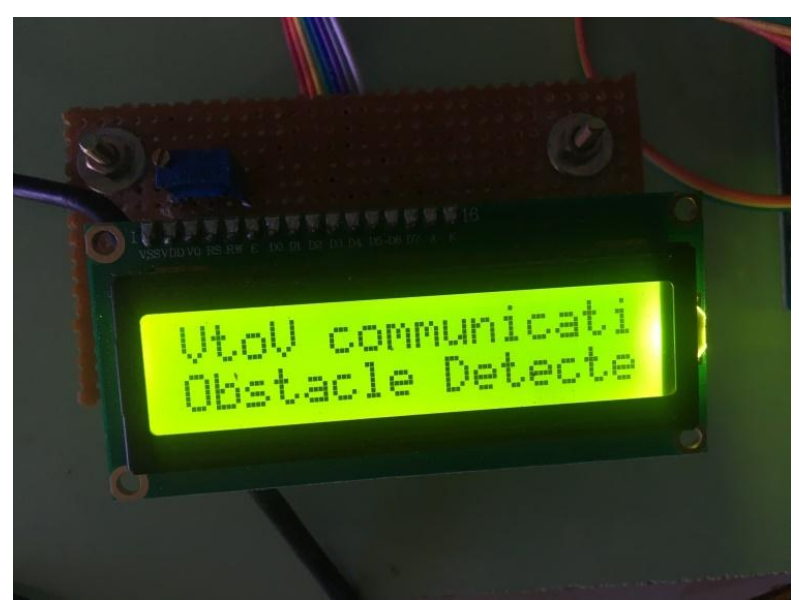

Fig7. LCD Showing Warning Message

The LED transmitter is modulated to greater extent and maximum speed is attained

\section{CONCLUSION AND FUTURE SCOPE}

Vehicle-to-Vehicle Communication is easy and simple to use. With the proposed system warning messages can be transmitted at faster rates that foster drivers to make strategic decision at faster rate which is vital in making strategic decision avoiding accidents and congestion. In future the project can be extended to communicate Vehicle-toInfrastructure for smart city.

\section{REFERENCES}

[1] Ambuj Kumar and Rajendea Prasad Nayak,"An Efficient Group-Basd Safety Message Transmission Protocol for
VANET",IEEE International Conference on communication and signal processing,April 3-5,2013 India, pp. $1-4$

[2] Sok-Ian Sou and Ozan.K.Tonguz"Enhancin VANET Connectvity through Roadside Units on Highway",IEEE transaction on Vehicular Technology, Vol. 60, No 8, October 2011, pp..3586-3602.

[3] Seh Chun Ng,Wuxiong Zhang, Yu Zhang ,Yang Yang "Analysis of Access and Connectivity Probablities in Vehicular Relay Network" IEEE journal ON selected Areas in Communication, Vol. 29,No.3,March 2011,pp. 140-150.

[4] A. Boukerche et al., "Vehicular Ad Hoc Networks: a new challenge for localization-based systems," Computer Communications, ScienceDirect, 2008, pp. 1-12.

[5] T. H. M. A. Y. K. K. K. Isamu Takai, "Optical Vehicleto-Vehicle Communication System Using LED Transmitter and Camera Receiver," IEEE Photonics Journal , Vol. 6, No. 5, October 2014, pp. 79025137902513.

[6] Yuichi Tanakat, Shinichirou Haruyamat, Masao Nakagawat "Wireless Optical Trasnsmissions with white colored LED for Wireless Home Links", Department of Electrical Engineering, Faculty of Science and Technology, Keio University.

[7] Samreen.N.Shaikh, Dr. S.R.Patil,“A Robust Broadcast Scheme for Vehicle to Vehicle communication",2016 Conference on Advances in Signal Processing(CASP) Cummins College of Engineering for Women, Pune. Jun $9-11,2016$ 